\title{
Large extra dimension effects through light-by-light scattering at the CERN LHC
}

\author{
Hao Sun ${ }^{\mathrm{a}}$ \\ Institute of Theoretical Physics, School of Physics and Optoelectronic Technology, Dalian University of Technology, \\ Dalian 116024, People's Republic of China
}

Received: 15 April 2014 / Accepted: 10 July 2014 / Published online: 2 August 2014

(C) The Author(s) 2014. This article is published with open access at Springerlink.com

\begin{abstract}
Observing light-by-light scattering at the large hadron collider (LHC) has received quite some attention and it is believed to be a clean and sensitive channel to possible new physics. In this paper, we study the diphoton production at the LHC via the process $p p \rightarrow p \gamma \gamma p \rightarrow p \gamma \gamma p$ through graviton exchange in the large extra dimension (LED) model. Typically, when we do the background analysis, we also study the double Pomeron exchange of $\gamma \gamma$ production. We compare its production in the quark-quark collision mode to the gluon-gluon collision mode and find that contributions from the gluon-gluon collision mode are comparable to the quark-quark one. Our result shows, for extra dimension $\delta=4$, with an integrated luminosity $\mathcal{L}=200 \mathrm{fb}^{-1}$ at the $14 \mathrm{TeV}$ LHC, that diphoton production through graviton exchange can probe the LED effects up to the scale $M_{S}=5.06(4.51,5.11) \mathrm{TeV}$ for the forward detector acceptance $\xi_{1}\left(\xi_{2}, \xi_{3}\right)$, respectively, where $0.0015<\xi_{1}<0.5$, $0.1<\xi_{2}<0.5$, and $0.0015<\xi_{3}<0.15$.
\end{abstract}

\section{Introduction}

The large hadron collider (LHC) at CERN generates high energetic proton-proton ( $p p$ ) collisions with a luminosity of $\mathcal{L}=10^{34} \mathrm{~cm}^{-2} \mathrm{~s}^{-1}$ and provides the opportunity to study very high energy physics. At such a high energy, most attention is usually paid to the central rapidity region where most of the particles are produced and where most of the high $p_{T}$ signal of new physics is expected. Indeed, the CDF collaboration has already observed such a kind of interesting phenomenon including the exclusive lepton pairs production $[1,2]$, photon-photon production [3], dijet production [4] and charmonium $(J / \psi)$ meson photoproduction [5], etc. Now, both ATLAS and CMS collaborations have programs of forward physics which are devoted to studies of high rapidity

a e-mail: haosun@mail.ustc.edu.cn; haosun@dlut.edu.cn regions with extra updated detectors located in a place nearly 100-400 m close to the interaction point [6-9]. Technical details of the ATLAS forward physics (AFP) projects can be found, for example, in Refs. [10,11]. The physics program of this new instrumentation covers interesting topics like elastic scattering, diffraction, low-x QCD, central exclusive production (CEP), and the photon-photon $(\gamma \gamma)$ interaction, the last two being the main motivation for the AFP project.

CEP is a class of processes in which the two interacting protons are not destroyed during the collision but survive into the final state with additional particle states. Protons of this kind are named intact or forward protons. This is a rare interaction and can take place through strong effects like Pomeron exchange or electromagnetic effects, i.e., via photon exchange. The kinematics of a forward proton is often described by means of the reduced energy loss $\xi$ :

$\xi=\frac{\Delta E}{E}=\frac{E-E^{\prime}}{E}$

where $E$ is the initial energy of the beam, $E^{\prime}$ is the energy after the interaction and $\Delta E$ is the energy that the proton lost in the interaction. For CEP, the simple approximate relation between the reduced energy losses of both protons ( $\xi_{1}$ and $\left.\xi_{2}\right)$ and the mass of the centrally produced system $M$ is

$M^{2}=s \xi_{1} \xi_{2}$

where $s=4 E^{2}$ is the square of the center-of-mass energy.

The simplest exclusive production is due to the exchange of two photons: $p p \rightarrow p \gamma \gamma p \rightarrow p X p$ where $X$ is the centrally produced system. In such production at the LHC, the invariant mass of the photons can span up to $1 \mathrm{TeV}$ scales, high enough to reach scales of possible new physics. In addition, the production of these processes mainly through the QED mechanisms which are well understood and their predictions have a very small uncertainty. These make the twophoton exchange physics particularly interesting. Therefore, we can use this kind of production mechanism to determine 
the luminosity at the LHC precisely [12], to study the interaction of electroweak bosons with over-constrained kinematics, to test the standard model (SM) at high energies or to study the new production channels that could appear, i.e., SUSY [13-18], anomalous gauge couplings [19-24], unparticle [25], and extra dimensions [26,27], etc.

Observing light-by-light scattering at the LHC has received quite some attention [28] and it is believed a clean and sensitive channel to possible new physics. In this paper, we study the diphoton signal from graviton exchange in the large extra dimension (LED) model via the main reaction $p p \rightarrow p \gamma \gamma p \rightarrow p G_{\mathrm{kk}} p \rightarrow p \gamma \gamma p$ where $G_{\mathrm{KK}}$ is the KK graviton in LED. A similar study has been performed in Ref. [27] where the authors study the diphoton signal in both the LED and Randall-Sundrum (RS) models and take $\gamma \gamma$ SM production as the corresponding background. In our study we also consider the double Pomeron exchange (DPE) production of the diphoton and present their cross section dependence on the energy loss of the proton $\xi$ and compare its production separately in the quark-quark collision mode to the gluon-gluon collision mode. Our paper is organized as follows: we build the calculation framework in Sect. 2 including a brief introduction to the central exclusive diphoton production and equivalent photon approximation (EPA), the general diphoton exchange process cross section and a brief introduction to the LED model. Section 3 is arranged to present the input parameters and numerical results of our study. Typically we present a discussion of DPE diphoton production. Finally we summarize our conclusions in the last section.

\section{Calculation framework}

\subsection{Central diphoton exchange at the LHC and EPA}

A generic diagram for the two-photon CEP $p p \rightarrow p \gamma \gamma p \rightarrow$ $p X p$ is presented in Fig. 1. When the invariant mass of system $X\left(M_{X}\right)$ produced in the two-photon process is not too small, we can factorize the amplitudes for the interaction $\gamma \gamma \rightarrow X$ and introduce equivalent photon fluxes which play a similar role to the parton density functions for the hadron interactions. This is indeed the basis of the EPA [29-31], and it allows us to calculate the proton cross section as the photon cross section plus two equivalent photon fluxes. Two quasireal photons emitted by each proton interact with each other to produce $X: \gamma \gamma \rightarrow X$. Deflected protons and their energy loss will be detected by the forward detectors, while the $X$ system will go to the central detector. In our case the $X$ system is the final state diphoton production. A final state photon with rapidity $|\eta|<2.5$ and some transverse momentum $p_{T}>(5-10) \mathrm{GeV}$ will be detected by the central detector. The initial exchanged photons emitted with small angles by



Fig. 1 A generic diagram for the two-photon exclusive production, $p p \rightarrow p \gamma \gamma p \rightarrow p X p$ at the CERN LHC. Two incoming protons are scattered quasi-elastically at very small angles. The produced system $X$ can be detected in the central detectors

the protons show a spectrum of virtuality $Q^{2}$ and the energy $E_{\gamma}$. This is described by the EPA mentioned above which differs from the point-like electron (positron) case by taking care of the electromagnetic form factors in the equivalent $\gamma$ spectrum and effective $\gamma$ luminosity:

$$
\begin{aligned}
& \frac{\mathrm{d} N_{\gamma}}{\mathrm{d} E_{\gamma} \mathrm{d} Q^{2}} \\
& =\frac{\alpha}{\pi} \frac{1}{E_{\gamma} Q^{2}}\left[\left(1-\frac{E_{\gamma}}{E}\right)\left(1-\frac{Q_{\min }^{2}}{Q^{2}}\right) F_{E}+\frac{E_{\gamma}^{2}}{2 E^{2}} F_{M}\right]
\end{aligned}
$$

with

$$
\begin{gathered}
Q_{\min }^{2}=\left(\frac{M_{\mathrm{inv}}^{2} E}{E-E_{\gamma}}-M_{p}^{2}\right) \frac{E_{\gamma}}{E}, \quad F_{E}=\frac{4 M_{p}^{2} G_{E}^{2}+Q^{2} G_{M}^{2}}{4 M_{p}^{2}+Q^{2}}, \\
G_{E}^{2}=\frac{G_{M}^{2}}{\mu_{p}^{2}}=\left(1+\frac{Q^{2}}{Q_{0}^{2}}\right)^{-4}, \quad F_{M}=G_{M}^{2}, \quad Q_{0}^{2}=0.71 \mathrm{GeV}^{2},
\end{gathered}
$$

where $\alpha$ is the fine-structure constant, $E$ is the energy of the incoming proton beam, which is related to the quasi-real photon energy by $E_{\gamma}=\xi E . M_{p}$ is the mass of the proton and $M_{\mathrm{inv}}$ is the invariant mass of the final state. $\mu_{p}^{2}=7.78$ is the magnetic moment of the proton. $F_{E}$ and $F_{M}$ are functions of the electric and magnetic form factors given in the dipole approximation.

We denote the general diphoton exchange processes at the LHC as

$p p \rightarrow p \gamma \gamma p \rightarrow p+i j k \cdots+p$

with $i, j, k, \ldots$ the centrally produced final state particles. The partonic cross section $\hat{\sigma}_{\gamma \gamma \rightarrow i j k \ldots}$ for the subprocess $\gamma \gamma \rightarrow i j k \ldots$ should be integrated over the photon spectrum and we can obtain the total cross section:

$$
\begin{aligned}
\sigma_{p p}= & \int_{\omega_{0}}^{\sqrt{s}} \hat{\sigma}_{\gamma \gamma \rightarrow i j k \ldots}\left(\omega_{\gamma \gamma}\right) \frac{\mathrm{d} L^{\gamma \gamma}}{\mathrm{d} \omega_{\gamma \gamma}} \mathrm{d} \omega_{\gamma \gamma} \\
= & \int_{\omega_{0}}^{\sqrt{s}} \hat{\sigma}_{\gamma \gamma \rightarrow j j}\left(\omega_{\gamma \gamma}\right) \int_{\omega_{\gamma \gamma}^{2} / s}^{1} \frac{2 \omega_{\gamma \gamma}}{x s} \int_{Q_{1, \min }^{2}}^{Q_{\max }^{2}} f_{1}\left(x, Q_{1}^{2}\right) \\
& \times \int_{Q_{2, \min }^{2}}^{Q_{\max }^{2}} f_{2}\left(\frac{\omega_{\gamma \gamma}^{2}}{x s}, Q_{2}^{2}\right) \mathrm{d} Q_{1}^{2} \mathrm{~d} Q_{2}^{2} \mathrm{~d} x \mathrm{~d} \omega_{\gamma \gamma}
\end{aligned}
$$


where $\sigma_{p p}$ is the total photon-photon cross section, $\omega_{\gamma \gamma}$ is the two-photon center-of-mass (c.m.s.) energy, or the invariant mass of the produced system $M, s=4 E^{2}, \omega_{0}$ is some initial energy and $Q_{\max }^{2}=2 \mathrm{GeV}^{2}$ is the maximum virtuality. $f=\frac{\mathrm{d} N}{\mathrm{~d} E_{\gamma} \mathrm{d} Q^{2}}$ is the $Q^{2}$-dependent relative luminosity spectrum presented in Eq. (3). We consider $E_{\gamma}=\xi E$ to be some fraction of the total beam energy, $M^{2}=\omega^{2}=$ $\xi_{1} \xi_{2} s=4 \xi_{1} \xi_{2} E^{2}$, and the forward detector acceptance satisfies $\xi_{\min } \leq \xi \leq \xi_{\max }$; we can also write the $\xi$-dependent cross section as

$$
\begin{aligned}
& \sigma_{p p}=\int_{\tau_{0}}^{1} \int_{\xi_{0}}^{\xi_{\max }} \int_{Q_{1, \min }^{2}}^{Q_{\max }^{2}} \int_{Q_{2, \min }^{2}}^{Q_{\max }^{2}} \hat{\sigma}_{\gamma \gamma \rightarrow i j k \ldots} \\
& \quad \times\left(\xi_{1} \xi_{2} s\right) f_{1}\left(\xi_{1}, Q_{1}^{2}\right) f_{2}\left(\xi_{2}, Q_{2}^{2}\right) \mathrm{d} Q_{1}^{2} \mathrm{~d} Q_{2}^{2} \mathrm{~d} \xi_{1} \mathrm{~d} \xi_{2},
\end{aligned}
$$

with $\tau_{0}=M_{\mathrm{inv}}^{2} / \mathrm{s}, M_{\mathrm{inv}}$ is the invariant mass of the $X$ system. $\xi_{0}=\operatorname{Max}\left(\xi_{\min }, \tau_{0} / \xi_{\max }\right)$. The cross section of the subprocess $\hat{\sigma}$ can be written as

$\hat{\sigma}=\int \frac{1}{\operatorname{avgfac}} \frac{\left|\mathcal{M}_{n}\left(\xi_{1} \xi_{2} s\right)\right|^{2}}{2 \hat{s}(2 \pi)^{3 n-4}} \mathrm{~d} \Phi_{n}$

where $\frac{1}{\text { avgfac }}$ is the factor of the spin-average factor, the coloraverage factor, and the identical particle factor (if there is any). $\left|\mathcal{M}_{n}\right|^{2}$ represents the squared $n$-particle matrix element and is divided by the flux factor $\left[2 \hat{s}(2 \pi)^{3 n-4}\right]$. The $n$-body phase space differential $\mathrm{d} \Phi_{n}$ and its integral $\Phi_{n}$ depend only on $\hat{s}$ and the particle masses $m_{i}$ due to Lorentz invariance:

$$
\begin{aligned}
& \Phi_{n}\left(\hat{s}, m_{1}, m_{2}, \ldots, m_{n}\right)=\int \mathrm{d} \Phi_{n}\left(\hat{s}, m_{1}, m_{2}, \ldots, m_{n}\right) \\
& =\int \delta^{4}\left(\left(p_{i}+p_{j}\right)-\sum_{k=1}^{n} p_{k}\right) \prod_{k=1}^{n} d^{4} p_{k} \delta\left(p_{k}^{2}-m_{k}^{2}\right) \Theta\left(p_{k}^{0}\right),
\end{aligned}
$$

with $i$ and $j$ denoting the incident particles and $k$ running over all outgoing particles.

\subsection{LED and light-light scattering through graviton exchange}

The hierarchy problem strongly suggests the existence of new physics beyond the $\mathrm{SM}$ at $\mathrm{TeV}$ scale. The idea that there exist extra dimensions (ED) which was first proposed by ArkaniHamed, Dimopoulos, and Dvali [32,33], might provide a solution to this problem. They proposed a scenario in which the SM field is constrained to the common $3+1$ spacetime dimensions ("brane"), while gravity is free to propagate throughout a larger multidimensional space $D=\delta+4$ ("bulk"). The picture of a massless graviton propagating in $D$ dimensions is equal to the picture that numerous massive Kaluza-Klein (KK) gravitons propagate in four dimensions. The fundamental Planck scale $M_{S}$ is related to the Planck mass scale $M_{\mathrm{Pl}}=G_{N}^{-1 / 2}=1.22 \times 10^{19} \mathrm{GeV}$ according to



(1)

(2)

(3)

Fig. 2 Feynman diagrams for light-light scattering of the diphoton production through graviton exchange in the LED model

the formula $M_{\mathrm{Pl}}^{2}=8 \pi M_{S}^{\delta+2} R^{\delta}$, where $R$ and $\delta$ are the size and number of the extra dimensions, respectively. If $R$ is large enough to make $M_{S}$ on the order of the electroweak symmetry breaking scale $(\sim 1 \mathrm{TeV})$, the hierarchy problem will be naturally solved, so this extra dimension model is called the LED model or the ADD model. Postulating $M_{S}$ to be $1 \mathrm{TeV}$, we get $R \sim 10^{13} \mathrm{~cm}$ for $\delta=1$, which is obviously ruled out since it would modify Newton's law of gravity at solarsystem distances; and we get $R \sim 1 \mathrm{~mm}$ for $\delta=2$, which is also ruled out by torsion-balance experiments [34]. When $\delta \geq 3$, where $R<1 \mathrm{~nm}$, it is possible to detect a graviton signal at high energy colliders.

At colliders, the exchange of a virtual KK graviton or the emission of a real KK mode could give rise to interesting phenomenological signals at $\mathrm{TeV}$ scale $[35,36]$. The virtual effects of the KK modes could lead to the enhancement of the cross section of pair productions in the processes, for example, dilepton, digauge boson $\left(\gamma \gamma, Z Z, W^{+} W^{-}\right)$, dijet, $t \bar{t}$ pair, HH pair [37-61], etc. The real emission of a KK mode could lead to large missing $E_{T}$ signals viz. mono jet, mono gauge boson $[35,36,62,63]$, etc. The CMS collaboration has performed a lot of research for LED on different final states at $\sqrt{s}=7 \mathrm{TeV}$ [64-66], and they set the most stringent lower limits to date to be $2.5 \mathrm{TeV}<M_{S}<3.8 \mathrm{TeV}$ by combining the diphoton, dimuon, and dielectron channels.

Now we give the analytical calculations of the process $\gamma \gamma \rightarrow \gamma \gamma$ at the LHC in the LED model. In our calculation we use the de Donder gauge. The relevant Feynman rules involving a graviton in the LED model can be found in Ref. [36]. We denote the process as

$\gamma\left(p_{1}\right)+\gamma\left(p_{2}\right) \rightarrow \gamma\left(p_{3}\right)+\gamma\left(p_{4}\right)$

where $p_{1}, p_{2}$ and $p_{3}, p_{4}$ represent the momenta of the incoming and outgoing particles, respectively. In Fig. 2 we display the Feynman diagrams for this process in the LED model. We see the diphoton productions going through $s-, t-, u$-channels of graviton exchange. The spin- 0 states only couple through the dilaton modes, which have no contribution to $\gamma \gamma$ processes. So we focus our study on the spin- 2 component of the KK states. The couplings between gravitons and SM particles are proportional to a constant named gravitational coupling $\kappa \equiv \sqrt{16 \pi G_{N}}$, which can be expressed in terms of the fundamental Planck scale $M_{S}$ and the size of the compactified space $R$ by 
$\kappa^{2} R^{\delta}=8 \pi(4 \pi)^{\delta / 2} \Gamma(\delta / 2) M_{S}^{-(\delta+2)}$.

In practical experiments, the contributions of the different Kaluza-Klein modes have to be summed up, so the propagator is proportional to $i /\left(s_{i j}-m_{\tilde{n}}^{2}\right)$, where $s_{i j}=\left(p_{i}+p_{j}\right)^{2}$ and $m_{\tilde{n}}$ is the mass of the KK state $\tilde{n}$. Thus, when the effects of all the KK states are taken together, the amplitude is proportional to $\sum_{\tilde{n}} \frac{i}{s_{i j}-m_{\tilde{n}}^{2}+i \epsilon}=D(s)$. If $\delta \geq 2$ this summation is formally divergent as $m_{\tilde{n}}$ becomes large. We assume that the distribution has a ultraviolet cutoff at $m_{\tilde{n}} \sim M_{S}$, where the underlying theory becomes manifest. Then $D(s)$ can be expressed as

$D(s)=\frac{1}{\kappa^{2}} \frac{8 \pi}{M_{S}{ }^{4}}\left(\frac{\sqrt{s}}{M_{S}}\right)^{\delta-2}\left[\pi+2 i I\left(M_{S} / \sqrt{s}\right)\right]$.

The imaginary part $I(\Lambda / \sqrt{s})$ is from the summation over the many non-resonant KK states and its expression can be found in Ref. [36]. Finally the KK graviton propagator after summing over the KK states is

$\tilde{G}_{\mathrm{KK}}^{\mu \nu \alpha \beta}=D(s)\left(\eta_{\mu \alpha} \eta_{\nu \beta}+\eta_{\mu \beta} \eta_{\nu \alpha}-\frac{2}{D-2} \eta_{\mu \nu} \eta_{\alpha \beta}\right)$.

Using the Feynman rules in the LED model and the propagator given by Eq. (13), we can get the amplitudes for the virtual KK graviton exchange diagrams in Fig. 2. For the formulas and details we refer to the two original calculations $[67,68]$. Inserting the amplitudes into Eqs. (7) and (8) we can obtain the total cross section.

\section{Numerical results}

\subsection{Input parameters and kinematic cuts}

We take the input parameters as $M_{p}=0.938272046 \mathrm{GeV}$, $\left.\alpha_{\mathrm{ew}}\left(m_{Z}^{2}\right)^{-1}\right|_{\overline{\mathrm{MS}}}=127.918, m_{Z}=91.1876 \mathrm{GeV}, m_{W}=$ $80.385 \mathrm{GeV}$ [69] and we have $\sin ^{2} \theta_{W}=1-\left(m_{W} / m_{Z}\right)^{2}=$ 0.222897 . Light fermion masses are needed in the SM oneloop $\gamma \gamma$ production and we take $m_{e}=0.510998910 \mathrm{MeV}$, $m_{\mu}=105.658367 \mathrm{MeV}, m_{\tau}=1,776.82 \mathrm{MeV}, m_{u}=m_{\mathrm{d}}=$ $53.8 \mathrm{MeV}, m_{c}=1.27 \mathrm{GeV}, m_{s}=101 \mathrm{MeV}, m_{b}=4.67$ $\mathrm{GeV}$. The top quark pole mass is set to be $m_{t}=173.5 \mathrm{GeV}$. The colliding energy in the proton-proton c.m.s. system is assumed to be $\sqrt{s}=14 \mathrm{TeV}$ at future LHC. We use FeynArts, FormCalc, and LoopTools (FFL) [70-73] packages to generate the amplitudes performing the numerical calculations. We adopt BASES $[74,75]$ to do the phase space integration. Based on the forward proton detectors to be installed by the CMS-TOTEM and the ATLAS collaborations we choose the detected acceptances to be

- CMS-TOTEM forward detectors with $0.0015<\xi_{1}<$ 0.5 ;
- CMS-TOTEM forward detectors with $0.1<\xi_{2}<0.5$;

- AFP-ATLAS forward detectors with $0.0015<\xi_{3}<$ 0.15 ,

which we simply refer to as $\xi_{1}, \xi_{2}$, and $\xi_{3}$, respectively. During the calculation we use $\xi_{1}$ in default unless otherwise stated. The survival probability in the $\gamma \gamma$ collision is taken to be 0.9 for central diphoton exchange and 0.03 for DPE processes [76].

In all the results present in our work, we impose a cut of pseudorapidity $|\eta|<2.5$ for the final state particles, since central detectors of the ATLAS and CMS have a pseudorapidity $|\eta|$ coverage of 2.5. The general acceptance cuts for all the signal and background events are

$\omega>300 \mathrm{GeV}, p_{T}^{\gamma}>20 \mathrm{GeV},|\eta|^{\gamma}<2.5, \Delta R(\gamma \gamma)>0.4$

where $\Delta R=\sqrt{\Delta \Phi^{2}+\Delta \eta^{2}}$ is the separation in the rapidityazimuth plane and $p_{T}$ is the transverse momentum of the photons.

\subsection{Background analysis}

Our signal topology is simply that of two photons in the final states excited by graviton exchange. The main background comes from two sources: one is the background coming from SM $p p \rightarrow p \gamma \gamma p \rightarrow p \gamma \gamma p$ production through one-loop contributions. The other comes from diffractive DPE [or Central Exclusive Diffractive (CED) production] of the diphoton.

Let us first consider the SM $\gamma \gamma \rightarrow \gamma \gamma$ one-loop production. Though there is no tree level contribution to $\gamma \gamma \rightarrow \gamma \gamma$, it is still important to include loop effects of its contributions. The precise determination of this cross section has a special importance to test the renormalization procedure of the parts of the SM containing $W$ gauge bosons. Moreover, as in our case, this loop process becomes a background for new physics searches through $\gamma \gamma$ scattering. One-loop diagrams involve charged fermions and the $W$ bosons rotate in loop. Here we include all these contributions and perform the calculation with the FFL package. The calculation can also be found in Ref. [27].

Now let us see the background contributions from DPE or CED productions. Different from Ref. [27] where one takes the SM $\gamma \gamma$ production as the only background, here we also consider the DPE backgrounds. However, their results are for $0.1<\xi_{2}<0.5$, where in this range, DPE indeed is small and not very important. But for the other choice of the forward detector acceptance it might be interesting to study them. In DPE, two colorless objects are emitted from both protons. Their partonic components are resolved and create a heavy mass object $X$ in the central detector in the PomeronPomeron interaction. The event is characterized by two rapidity gaps between the central object and the protons. Through the exchange of two Pomerons a dijet system, a diphoton 
system, a WW and ZZ pair, or a Drell-Yan pair can be created for instance. The DPE production cross section can be obtained within the factorized Ingelman-Schlein [77]. In the Ingelman-Schlein model, one assumes that the Pomeron has a well-defined partonic structure and that the hard process takes place in Pomeron-proton or proton-Pomeron (single diffraction) or Pomeron-Pomeron (central diffraction) processes. These processes are described in terms of the proton "diffractive" parton distribution functions (DPDFs). Similar to the parton distribution functions, the DPDFs are also obtained from Deep Inelastic Scattering experiments [78,79]. The difference is that they contain a dependence on additional variables describing the proton kinematics: the relative energy loss $\xi$ and four-momentum transfer $t$, which we introduce as

$$
\begin{aligned}
g^{D}\left(x, \mu^{2}\right) & =\int \mathrm{d} x_{\mathbf{P}} \mathrm{d} \beta \delta\left(x-x_{\mathbf{P}} \beta\right) g_{\mathbf{P}}\left(\beta, \mu^{2}\right) f_{\mathbf{P}}\left(x_{\mathbf{P}}\right) \\
& =\int_{x}^{1} \frac{\mathrm{d} x_{\mathbf{P}}}{x_{\mathbf{P}}} f_{\mathbf{P}}\left(x_{\mathbf{P}}\right) g_{\mathbf{P}}\left(\frac{x}{x_{\mathbf{P}}}, \mu^{2}\right)
\end{aligned}
$$

where $g^{D}$ denotes either the quark or the gluon distributions with $D$ referring to "diffractive". $f_{\mathbf{P}}\left(x_{\mathbf{P}}\right)$ is the flux of the Pomerons and is expressed as

$$
f_{\mathbf{P}}\left(x_{\mathbf{P}}\right)=\int_{t_{\min }}^{t_{\max }} f\left(x_{\mathbf{P}}, t\right) \mathrm{d} t
$$

with $t_{\min }$ and $t_{\max }$ being kinematic boundaries. $g_{\mathbf{P}}\left(\beta, \mu^{2}\right)$ is the partonic structure of the Pomeron. $x_{\mathbf{P}}$ here is the fraction of the proton momentum carried by the Pomeron corresponding to the relative energy loss $\xi$ and $\beta$ is the fraction of the Pomeron momentum carried by the struck parton. Both Pomeron flux factors $f\left(x_{\mathbf{P}}, t\right)$ as well as quark and gluon distributions in the Pomeron $g_{\mathbf{P}}\left(\beta, \mu^{2}\right)$ were taken from the $\mathrm{H} 1$ collaboration analysis of the diffractive structure function at HERA $[78,79]$. Therefore, the final forward detector acceptance's $\xi$-dependent convolution integral for the DPE production is given by

$$
\begin{aligned}
\sigma & =\sum_{i j=q \bar{q}, g g} \int_{\frac{M_{\text {inv }}}{\sqrt{s}}}^{\xi_{\max }} 2 z \mathrm{~d} z \int_{\operatorname{Max}\left(\frac{z^{2}}{\xi_{\max }}, \xi_{\min }\right)}^{\operatorname{Min}\left(\xi_{\max }, \frac{z^{2}}{\xi_{\min }}\right)} \frac{\mathrm{d} x_{1}}{x_{1}} \\
& \times \int_{x_{1}}^{1} \frac{\mathrm{d} x_{\mathbf{P}_{i}}}{x_{\mathbf{P}_{i}}} f_{\mathbf{P}_{i}}\left(x_{\mathbf{P}_{i}}\right) g_{\mathbf{P}_{i}}\left(\frac{x_{1}}{x_{\mathbf{P}_{i}}}, \mu^{2}\right) \int_{\frac{z^{2}}{x_{1}}}^{1} \frac{\mathrm{d} x_{\mathbf{P}_{j}}}{x_{\mathbf{P}_{j}}} f_{\mathbf{P}_{j}}\left(x_{\mathbf{P}_{j}}\right) g_{\mathbf{P}_{j}} \\
& \times\left(\frac{z^{2}}{x_{1} x_{\mathbf{P}_{j}}}, \mu^{2}\right) \hat{\sigma}\left(i j \rightarrow \gamma \gamma, \hat{s}=z^{2} s, \mu\right) .
\end{aligned}
$$

Diffraction usually dominates for $\xi<0.05$, which means that we can replace $\operatorname{Min}\left(\xi_{\max }, \frac{z^{2}}{\xi_{\min }}\right)$ by 0.05 directly. We check this and find the difference is quite small, as expected. The DPE production cross section should be multiplied by the gap survival probability $S=0.03$ for LHC. This is different for



(1)

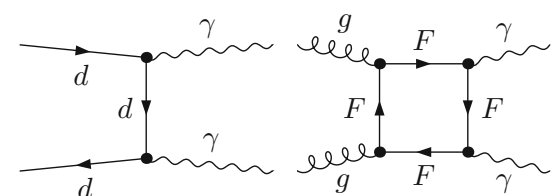

(2)
(3)
Fig. 3 Feynman diagrams for DPE contributions in the $u \bar{u}$, the $d \bar{d}$, and the $g g$ collision modes

$\gamma \gamma$ exchange where $S=0.9$ and single diffractive production with $S=0.6$ [76].

We separate the DPE background contributions into three different parts based on different parton collision modes, including $u \bar{u}, d \bar{d}$, and $g g$. Part of the Feynman diagrams are presented in Fig. 3. Other diagrams include the change of the loop arrow and cross change of the legs, which are similar and thus are not shown. $F$ means the class of fermions. $u \bar{u}$ and $d \bar{d}$ contributions are considered at tree level. Typically, we also include the $g g$ collision mode contribution where there are no tree level diagrams but we start from gluon-gluon fusion at one-loop level through fermion loops. We do this because we want to see how large their contributions will be, especially the $g g$ collision modes, since usually one does not consider them. Indeed we find that the $g g$ contribution is comparable with the quark-quark collision mode even though they contribute through loops. See our discussion below.

Before selecting our event samples for diphoton production, we order the two photons on the basis of their transverse momenta, i.e., $p_{T}^{\gamma_{1}} \geq p_{T}^{\gamma_{2}} \cdot p_{T}^{\gamma_{1}}$ is named the leading photon and simply is denoted as $p_{T}^{\gamma}$ in the following text and figures. Figure 4 presents the diphoton background productions. Different parton collision modes are considered in order to clearly compare their contributions. At this moment, no survival probability factor is taken into account. Dashed, shortdashed, dotted, and dashed-dotted lines refer to the DPE $u \bar{u}$ collision mode, the $d \bar{d}$ collision mode, the $g g$ collision mode, and their sum, respectively. Here $u$ includes both the $u$-quark and the $c$-quark, and $d$ refers to $d-, s$-, and $b$-quarks. We can see that the $u \bar{u}$ collision mode is the largest one. As regards the $g g$ collision mode, though there is no tree level contribution and it only appears at the loop level, its contribution is comparable with the quark collision. Both are larger than the $d \bar{d}$ collision mode. When no survival probability factor is considered, their contributions are much larger than the SM $\gamma \gamma$-loop contributions. But they become comparable when the survival probability factor is taken into account. More details can be found in Table 1. After considering these, the survival probability factor contributions of DPE are only three times over the SM loop contribution. The total backgrounds before and after taking into account the survival probability factor are 14.2846 and $0.5516 \mathrm{fb}$, respectively. 


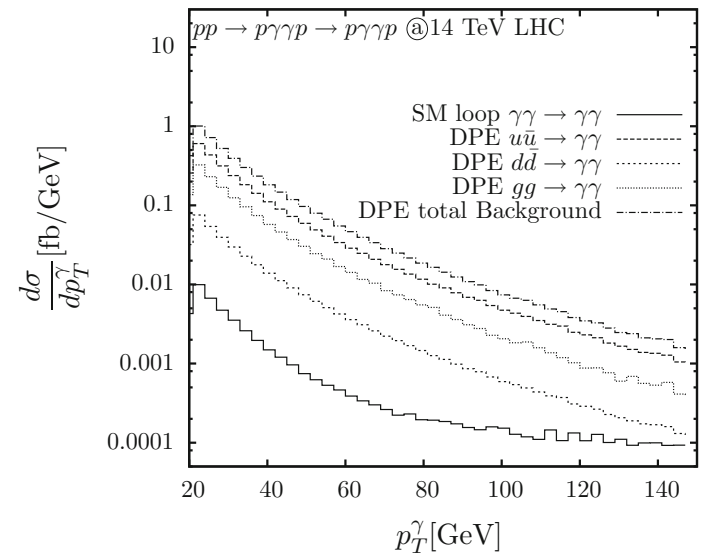

Fig. 4 The transverse momentum $\left(p_{T}\right)$ and rapidity ( $y$ ) distribution of a leading photon for the background production of $p p \rightarrow p \gamma \gamma p \rightarrow$ $p \gamma \gamma p$, on the basis of their transverse momentum $p_{T}^{\gamma_{1}} \geq p_{T}^{\gamma_{2}}$. The kinematic cuts are considered and no survival probability factor is taken

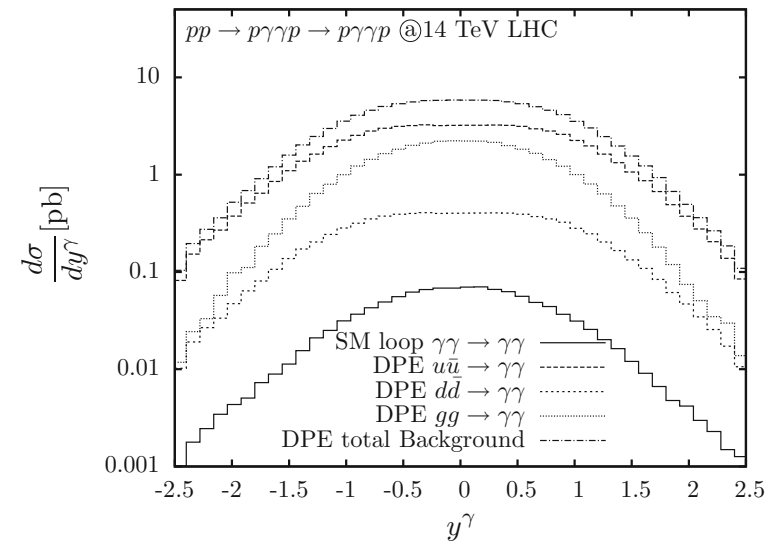

into account. The solid curve presents the SM $\gamma \gamma$-loop contribution. Dashed, short-dashed, dotted, and dashed-dotted lines refer to the DPE $u \bar{u}$ collision mode, the $d \bar{d}$ collision mode, the $g g$ collision mode, and their sum, respectively. Here $0.0015<\xi_{1}<0.5$

Table 1 Background contribution for diphoton production in different parton collision modes before and after taking into account the survival probability factor

\begin{tabular}{|c|c|c|c|c|c|}
\hline \multirow{2}{*}{$\begin{array}{l}\sigma(\mathrm{fb}) \\
\left(S_{\mathrm{DPE}}, S_{\gamma \gamma}\right)\end{array}$} & \multicolumn{5}{|c|}{ Background contribution for diphoton production } \\
\hline & $u \bar{u}$ & $d \bar{d}$ & $g g$ & $\mathrm{SM}_{\text {loop }}$ & Total \\
\hline$(1,1)$ & 8.5792 & 1.0724 & 4.49159 & 0.1415 & 14.2846 \\
\hline$(0.03,0.9)$ & 0.2574 & 0.0322 & 0.1347 & 0.1273 & 0.5516 \\
\hline
\end{tabular}

\subsection{Signal boundary at future LHC}

We are interested in the $p_{T}^{\gamma}$ distributions, which are displayed in Fig. 5. Both the transverse momentum of signal and the total background distribution of leading photon are presented. The range of the transverse momentum is displayed up to $1 \mathrm{TeV}$. The kinematic cuts and survival probability factors $S_{\mathrm{DPE}}=0.03$ and $S_{\gamma \gamma}=0.9$ are taken into account. The solid line refers to the total background including the sum of the SM $\gamma \gamma \rightarrow \gamma \gamma$-loop contribution and the DPE diphoton contributions. It is interesting that most of their contributions are in the low $p_{T}$ range. One may observe that, with a $p_{T}^{\gamma}$ cut larger than $\sim 300 \mathrm{GeV}$, all the background contributions can be reduced, while leaving the signal changes slight. Using this feature the light-by-light scattering of the diphoton central production at the LHC can be almost background free and can be detected precisely. If, in this case, a new physics signal appears mainly in the high transverse momentum range, light-by-light scattering can be very sensitive to it. This is exactly the LED effects we studied here. In this case, in the high $p_{T}^{\gamma}$ region, the LED effects dominate the signal distribution since more KK modes contribute with the increase of $p_{T}$. We use dashed, dotted, and dashed-dotted lines to refer to the LED signal with $M_{S}$ equal 3.5, 4.5 and $5.5 \mathrm{TeV}$, respectively. Just to note, in the following data analysis, we do not take the cut $p_{T}^{\gamma}>300 \mathrm{GeV}$. Nevertheless, we can still use

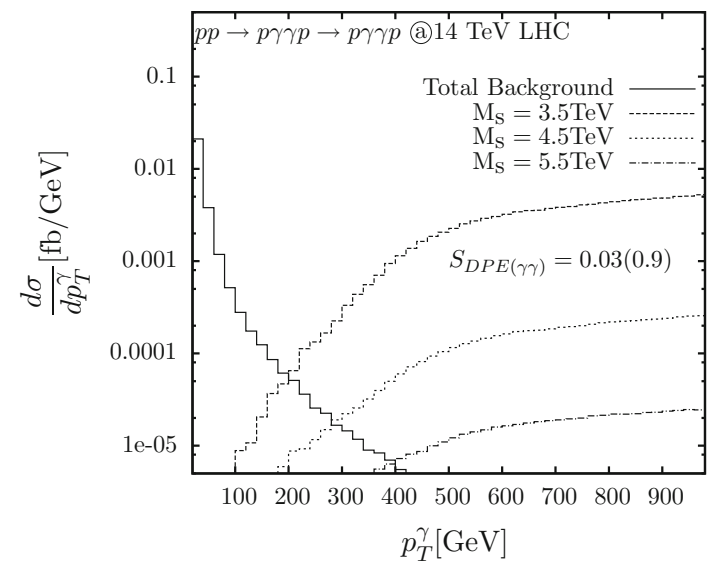

Fig. 5 The signal and total background transverse momentum $\left(p_{T}^{\gamma}\right)$ distribution of leading photon for the process $p p \rightarrow p \gamma \gamma p \rightarrow p \gamma \gamma p$, on the basis of their transverse momentum $p_{T}^{\gamma_{1}} \geq p_{T}^{\gamma_{2}}$ with $M_{S}=$ $3.5,4.5,5.5 \mathrm{TeV}, \sqrt{s}=14 \mathrm{TeV}$, and $\delta=4$. The high transverse momentum range includes up to $1 \mathrm{TeV}$. The kinematic cuts and survival probability factors $S_{\mathrm{DPE}}=0.03$ and $S_{\gamma \gamma}=0.9$ are taken into account

diphoton production to test the LED effects up to high energy scales.

In Fig. 6 we present the dependence of the cross section on the energy scale $M_{S}$ with $0.0015<\xi_{1}<0.5$, $0.1<\xi_{2}<0.5$, and $0.0015<\xi_{3}<0.15$, corresponding to 




Fig. 6 The total (signal plus background) cross sections for the process $p p \rightarrow p \gamma \gamma p \rightarrow p \gamma \gamma p$ in the SM and LED model as a function of $M_{S}$ with $0.0015<\xi_{1}<0.5,0.1<\xi_{2}<0.5,0.0015<\xi_{3}<0.15$, and $\delta=4$. The kinematic cuts and survival probability factors $S_{\mathrm{DPE}}=0.03$ and $S_{\gamma \gamma}=0.9$ are taken into account

solid, dashed, and dotted lines, respectively. We present the curves for the cross sections with the extra dimension $\delta=4$. The curves present the signal plus its corresponding background. The kinematic cuts in Eq. (14) are considered and the survival probability factors $S_{\mathrm{DPE}}=0.03$ and $S_{\gamma \gamma}=0.9$ for central production at the LHC are taken into account. It is clear that, for a given value of $\delta$, the cross section decreases rapidly with the increment of $M_{S}$, and finally approaches its corresponding background results. We can see that the cross sections of $\xi_{1}$ and $\xi_{3}$ are very close to each other, and both are larger than the results of $\xi_{3}$, two orders of magnitude larger, both for the signal and the background.

To analyze the potential of the $\gamma \gamma$ collision at the high energy LHC to probe the diphoton signal through LED effects, we define the statistical significance (SS) of the signal:

$\mathrm{SS}=\frac{\left|\sigma_{S+B}-\sigma_{B}\right|}{\sqrt{\sigma_{B}}} \sqrt{\mathcal{L}}$

where $\mathcal{L}$ is the integrated luminosity of the $\gamma \gamma$ option of the LHC. In the $\mathcal{L}-M_{S}$ parameter space, we present the plots for the $3 \sigma$ and $5 \sigma$ deviations of the signal from the total backgrounds. We display our results for the forward detector acceptance chosen to be $0.0015<\xi_{1}<0.5,0.1<\xi_{2}<0.5$, and $0.0015<\xi_{3}<0.15$ in Fig. 7; see the first, the second, and the third panel, respectively. The c.m.s. energy of the LHC collider is $\sqrt{s}=14 \mathrm{TeV}$. The extra dimension is set to be $\delta=4$. Concerning the criteria, for a statistical significance of $\mathrm{SS}>5$, with an integrated luminosity $\mathcal{L}=100 \mathrm{fb}^{-1}, p p \rightarrow p \gamma \gamma p \rightarrow p \gamma \gamma p$ production through graviton exchange can probe the LED effects up to $M_{S}=$ $4.95(4.38,4.96) \mathrm{TeV}$ for $\xi_{1}\left(\xi_{2}, \xi_{3}\right)$. With an integrated luminosity $\mathcal{L}=200 \mathrm{fb}^{-1}$ one can probe the LED effects up to


Fig. 7 The $M_{S}$ bounds for $3 \sigma$ (solid lines) and $5 \sigma$ (dashed lines) deviations from the total backgrounds as functions of the luminosity $\mathcal{L}$ with extra dimensions $\delta=4$ for different choices of the forward detector acceptance $\xi$

the scale $M_{S}=5.06(4.51,5.11) \mathrm{TeV}$ for $\xi_{1}\left(\xi_{2}, \xi_{3}\right)$, respectively. The current limits on $M_{S}$ of the LEDs are about 3$5 \mathrm{TeV}$ from recent ATLAS and CMS results. Our results show that the process we considered can probe the LED effects only moderately above the current experimentally limits. The limits we obtained are indeed worse than that obtained by other conventional approaches like dijet production signals at the 
LHC, which give a reach of about $10 \mathrm{TeV}$ for $M_{S}$. Moreover, this is a little worse than or comparable to the diphoton production at the LHC which can extend this search to 5.3-6.7 TeV and the Drell-Yan process which can drive the search up to about $5.8 \mathrm{TeV}$. However, the limits we obtained are better than the results obtained by monojet signals (i.e., $4.5 ; 3.3 \mathrm{TeV}$ for $n=2 ; 6$ ), or a similar photon-induced study at the LHC using the subprocess $\gamma \gamma \rightarrow \ell^{+} \ell^{-}$[26].

\section{Summary}

A lot of theoretical work has been done to study the LED effects. Even if a process can be traced back to a definite set of operators, it is rarely the case that a particular collider signature can be traced back to a unique process. For this reason many different, complementary measurements are usually required to uncover the underlying new physics processes. Observing light-by-light scattering at the LHC has recently received much more attention [28]. It is believed to be a clean and sensitive channel to possible new physics. In this paper, we calculate the diphoton production at the LHC via the process $p p \rightarrow p \gamma \gamma p \rightarrow p \gamma \gamma p$ in the SM and LED. Typically, when we do a background analysis, we also study the DPE of $\gamma \gamma$ production. We present their cross section dependence on the energy loss of the proton $\xi$ and compare its production separately in the quark-quark collision mode to the gluon-gluon collision mode. We conclude that the contribution from the gluon-gluon collision mode are comparable to the quark-quark collision mode. A $p_{T}^{\gamma}>300 \mathrm{GeV}$ cut can suppress the DPE background efficiently. However, if there are no kinematic cuts taken into account, especially in the low $\xi$ range, they should better be studied and considered. Finally, we present the $M_{S}$ bounds for the $3 \sigma$ and $5 \sigma$ deviations from the total backgrounds as functions of the luminosity $\mathcal{L}$ with extra dimensions $\delta=4$ for different choices of the forward detector acceptance $\xi$. Concerning the criteria, for a statistical significance of SS $>5$, with an integrated luminosity $\mathcal{L}=200 \mathrm{fb}^{-1}, p p \rightarrow p \gamma \gamma p \rightarrow p \gamma \gamma p$ production through graviton exchange can probe the LED effects up to the scale $M_{S}=5.06(4.51,5.11) \mathrm{TeV}$ for $\xi_{1}\left(\xi_{2}, \xi_{3}\right)$, respectively, where $0.0015<\xi_{1}<0.5,0.1<\xi_{2}<0.5$, and $0.0015<\xi_{3}<0.15$.

Acknowledgments Project supported by the National Natural Science Foundation of China (No. 11205070), Shandong Province Natural Science Foundation (No. ZR2012AQ017) and by the Fundamental Research Funds for the Central Universities (No. DUT13RC(3)30).

Open Access This article is distributed under the terms of the Creative Commons Attribution License which permits any use, distribution, and reproduction in any medium, provided the original author(s) and the source are credited.

Funded by $\mathrm{SCOAP}^{3}$ / License Version CC BY 4.0.

\section{References}

1. Abulencia, A., et al.: (CDF Collaboration), Observation of exclusive electron-positron production in hadron-hadron collisions. Phys. Rev. Lett. 9(8), 112001 (2007). hep-ex/0611040

2. Aaltonen, T. et al.: (CDF Collaboration), Search for exclusive zboson production and observation of high-mass $p \bar{p} \rightarrow p \gamma \gamma \bar{p} \rightarrow$ $p l^{+} l^{-} \bar{p}$ events in $p \bar{p}$ collisions at $\sqrt{s}=1.96 \mathrm{TeV}$. Phys. Rev. Lett. 1(02), 222002 (2009). arXiv:0902.2816

3. Aaltonen, T., et al.: (CDF Collaboration), Search for exclusive $\gamma \gamma$ production in hadron-hadron collisions. Phys. Rev. Lett. 9(9), 242002 (2007). arXiv:0707.2374

4. Aaltonen, T., et al.: (CDF Collaboration), Observation of exclusive dijet production at the Fermilab Tevatron $\bar{p} p$ collider. Phys. Rev. D 7(7), 052004 (2008). arXiv:0712.0604

5. Aaltonen, T., et al.: (cDF collaboration), Observation of exclusive charmonium production and $\gamma \gamma \rightarrow \mu^{+} \mu^{-}$in $p \bar{p}$ collisions at $\sqrt{s}=1.96$ TeV. Phys. Rev. Lett. 1(02), 242001 (2009). arXiv:0902.1271

6. Tasevsky, M.: Diffractive physics program in ATLAS experiment, Measuring central exclusive processes at LHC. Nucl. Phys. Proc. Suppl. 1(79-180), 187-195 (2008). arXiv:0910.5205

7. Royon, C.: (RP220 Collaboration), Project to install roman pot detectors at $220 \mathrm{~m}$ in ATLAS. arXiv:0706.1796

8. Albrow, M.G., et al.: FP420: An \& proposal to investigate the feasibility of installing proton tagging detectors in the 420-m region at LHC, CERN-LHCC-2005-025 (2005)

9. Cox, B.E.: (FP420 R and D Collaboration), The FP420 R\& D Project at the LHC. hep-ph/0609209

10. Royon, C.: The ATLAS forward physics project. arXiv: 1302.0623

11. Staszewski, R.: ATLAS Collaboration. The AFP Project. Acta Physica Polonica B(42), 1615 (2011). arXiv:1104.1858

12. M.W. Krasny, J. Chwastowski, K. Slowikowski, Luminosity measurement method for LHC: the theoretical precision and the experimental challenges. Nucl. Instrum. Methods A584, 42-52 (2008)

13. Schul, N., Piotrzkowski, K.: Detection of two-photon exclusive production of supersymmetric pairs at the LHC. Nucl. Phys. Proc. Suppl. 1(79-180), 289-297 (2008). arXiv:0806.1097

14. Piotrzkowski, K., Schul, N.: Two-photon exclusive production of supersymmetric pairs at the LHC. AIP Conf. Proc. 1(200), 434-437 (2010). arXiv:0910.0202

15. Heinemeyer, S., Khoze, V.A., Ryskin, M.G., Stirling, W.J., Tasevsky, M., Weiglein, G.: Studying the MSSM Higgs sector by forward proton tagging at the LHC. Eur. Phys. J. C 5(3), 231-256 (2008). arXiv:0708.3052

16. Central Exclusive Diffractive MSSM Higgs-Boson production at the LHC. J. Phys. Conf. Ser. 1(10), 072016 (2008). arXiv:0801.1974

17. Heinemeyer, S., Khoze, V.A., Ryskin, M.G., Tasevsky, M., Weiglein, G., Higgs, B.S.M.: Physics in the exclusive forward proton mode at the LHC. Eur. Phys. J. C 7(1), 1649 (2011). arXiv: 1012.5007

18. Tasevsky, M.: Exclusive MSSM Higgs production at the LHC after Run I. Eur. Phys. J. C 73, 2672 (2013). arXiv: 1309.7772

19. Pierzchala, T., Piotrzkowski, K.: Sensitivity to anomalous quartic gauge couplings in photon-photon interactions at the LHC. Nucl. Phys. Proc. Suppl. 1(79-180), 257-264 (2008). arXiv:0807.1121

20. Kepka, O., Royon, C.: Anomalous $W W \gamma$ coupling in photoninduced processes using forward detectors at the CERN LHC. Phys. Rev. D 78, 073005 (2008). arXiv:0808.0322

21. Royon, C., Chapon, E., Kepka, O.: Anomalous trilinear and quartic WW gamma, WW gamma gamma, ZZ gamma and ZZ gamma gamma couplings in photon induced processes at the LHC. PoS EPS-HEP2009. 3, 80 (2009). arXiv:0909.5237 
22. Chapon, E., Royon, C., Kepka, O.: Anomalous quartic $W W \gamma \gamma$, $Z Z \gamma \gamma$, and trilinear $W W \gamma$ couplings in two-photon processes at high luminosity at the LHC. Phys. Rev. D 81, 074003 (2010). arXiv:0807.1121

23. Gupta, R.S.: Probing quartic neutral gauge boson couplings using diffractive photon fusion at the LHC. Phys. Rev. D 85, 014006 (2012). arXiv: 1111.3354

24. Fichet, S., von Gersdorff, G., Kepka, O., Lenzi, B., Royon, C., Saimpert, M.: Probing new physics in diphoton production with proton tagging at the Large Hadron Collider. arXiv:1312.5153

25. Sahin, I., Inan, S.C.: Probe of unparticles at the LHC in exclusive two lepton and two photon production via photon-photon fusion. JHEP 0909, 069 (2009). arXiv:0907.3290

26. Atag, S., Inan, S.C., Sahin, I.: Extra dimensions in photon-induced two lepton final states at the CERN-LHC. Phys. Rev. D 80, 075009 (2009). arXiv:0904.2687

27. Atag, S., Inan, S.C., Sahin, I.: Extra dimensions in $\gamma \gamma \rightarrow \gamma \gamma$ process at the CERN-LHC. JHEP 09, 042 (2010). arXiv: 1005.4792

28. D. d'Enterria, G.G. da Silveira, Observing light-by-light scattering at the Large Hadron Collider. Phys. Rev. Lett. 111, 080405 (2013)

29. V.M. Budnev, I.F. Ginzburg, G.V. Meledin, V.G. Serbo, The twophoton particle production mechanism. Physical problems. Applications. Equivalent photon approximation. Phys. Rep. 15, 181 (1975)

30. Baur, G., Hencken, K, Trautmann, D., Sadowsky, S., Kharlov, Y.: Coherent $\gamma \gamma$ and $\gamma A$ interactions in very peripheral collisions at relativistic ion colliders. Phys. Rep. 364, 359 (2002). hep-ph/0112211

31. Piotrzkowski, K.: Tagging two-photon production at the CERN Large Hadron Collider. Phys. Rev. D 63, 071502 (2001). hep-ex/0009065

32. Arkani-Hamed, N., Dimopoulos, S., Dvali, G.R.: The hierarchy problem and new dimensions at a millimeter. Phys. Lett. B 429, 263 (1998). hep-ph/9803315

33. Arkani-Hamed, N., Dimopoulos, S., Dvali, G.R.: Phenomenology, astrophysics and cosmology of theories with submillimeter dimensions and TeV scale quantum gravity. Phys. Rev. D 59, 086004 (1999). hep-ph/9807344

34. Kapner, D.J., Cook, T.S., Adelberger, E.G., Gundlach, J.H., Heckel, B.R., Hoyle, C.D., Swanson, H.E.: Tests of the gravitational inverse-square law below the dark-energy length scale. Phys. Rev. Lett. 98, 021101 (2007). hep-ph/0611184

35. Giudice, G.F., Rattazzi, R., Wells, J.D.: Quantum gravity and extra dimensions at high-energy colliders. Nucl. Phys. B 544, 3-38 (1999). hep-ph/9811291

36. T. Han, J.D. Lykken, R.-J. Zhang, On Kaluza-Klein states from large extra dimensions. Phys. Rev. D 59, 105006 (1999)

37. J.L. Hewett, Phys. Rev. Lett. 82, 4765 (1999)

38. P. Mathews, V. Ravindran, K. Sridhar, W.L. van Neerven, Nucl. Phys. B 713, 333 (2005)

39. Mathews, P., Ravindran, V.: Nucl. Phys. B 753, 1 (2006)

40. M.C. Kumar, P. Mathews, V. Ravindran, Eur. Phys. J. C 49, 599 (2007)

41. O.J.P. Eboli, T. Han, M.B. Magro, Phys. Rev. D 61, 094007 (2000)

42. K.M. Cheung, G.L. Landsberg, Phys. Rev. D 62, 076003 (2000)

43. M.C. Kumar, P. Mathews, V. Ravindran, A. Tripathi, Phys. Lett. B 672, 45 (2009)

44. Nucl. Phys. B 818, 28 (2009)

45. Kober, M., Koch, B., Bleicher, M.: Phys. Rev. D 76, 125001 (2007). arXiv:0708.2368

46. Gao, J., Li, C.S., Gao, X., Zhang, J.J.: Phys. Rev. D 80 , 016008 (2009). arXiv:0903.2551

47. N. Agarwal, V. Ravindran, V.K. Tiwari, A. Tripathi, Nucl. Phys. B 830, 248 (2010)

48. Z.U. Usubov, I.A. Minashvili, Phys. Part. Nucl. Lett. 3, 153 (2006)

49. Pisma Fiz. Elem. Chast. Atom. Yadra 3, 24 (2006)
50. Lee, K.Y., Song, H.S., Song, J.-H.: Phys. Lett. B 464, 82 (1999). hep-ph/9904355

51. N. Agarwal, V. Ravindran, V.K. Tiwari, A. Tripathi, Phys. Rev. D 82, 036001 (2010)

52. Yu-Ming B., Lei, G., Xiao-Zhou, L., Wen-Gan, M., Ren-You, Z.: Phys. Rev. D 85, 016008 (2012). arXiv:1112.4894

53. P. Mathews, S. Raychaudhuri, K. Sridhar, Phys. Lett. B 450, 343 (1999)

54. JHEP 0007, 008 (2000)

55. Lee, K.Y., Song, H.S., Song, J.-H., Yu, C.: Phys. Rev. D 60, 093002 (1999). hep-ph/9905227

56. Lee, K.Y., Park, S.C., Song, H.S., Song, J.-H., Yu, C.: Phys. Rev. D 61, 074005 (2000) (hep-ph/0105326). hep-ph/9910466

57. S.C. Inan, A.A. Billur, Phys. Rev. D 84, 095002 (2011)

58. C.S. Kim, K.Y. Lee, J. Song, Phys. Rev. D 64, 015009 (2001)

59. Datta, A., Gabrielli, E., Mele, B.: JHEP 0310, 003 (2003). hep-ph/0303259

60. H. Sun, Y.-J. Zhou, H. Chen, Eur. Phys. J. C 72, 2011 (2012)

61. H. Sun, Y.-J. Zhou, JHEP 11, 127 (2012)

62. Mirabelli, E.A., Perelstein, M., Peskin, M.E., Phys. Rev. Lett. 82, 2236 (1999). hep-ph/9811337

63. Cheung, K.-M., Keung, W.-Y.: Phys. Rev. D 60, 112003 (1999). hep-ph/9903294

64. C. Collaboration [CMS Collaboration]. arXiv:1204.0821 [hep-ex]

65. Chatrchyan, S. et al.: [CMS Collaboration], Phys. Lett. B 711, 15 (2012). arXiv:1202.3827 [hep-ex]

66. Chatrchyan, S., et al.: [CMS Collaboration], arXiv:1112.0688 [hepex]

67. Cheung, K.: Diphoton signals for low scale gravity in extra dimensions. Phys. Rev. D 61, 015005 (2000). hep-ph/9904266

68. Davoudiasl, H.: Gamma gamma $\rightarrow$ gamma gamma as a test of weak scale quantum gravity at the NLC. Phys. Rev. D 60, 084022 (1999). hep-ph/9904425

69. Beringer, J., et al.: (Particle Data Group), Review of Particle Physics (RPP). Phys. Rev. D 86, 010001 (2012)

70. Hahn, T.: Generating Feynman diagrams and amplitudes with FeynArts 3. Comput. Phys. Commun. 140, 418-431 (2001). hep-ph/0012260

71. Hahn, T.: Automatic loop calculations with FeynArts, FormCalc, and LoopTools. Nucl. Phys. Proc. Suppl. 89, 231-236 (2000). hep-ph/0005029

72. Agrawal, S., Hahn, T., Mirabella, E.: FormCalc 7. J. Phys. Conf. Ser. 368, 012054 (2012). arXiv: 1112.0124

73. Hahn, T., Perez-Victoria, M.: Automatized one loop calculations in four-dimensions and D-dimensions. Comput. Phys. Commun. 118, 153-165 (1999). hep-ph/9807565

74. S. Kawabata, A new version of the multi-dimensional integration and event generation package BASES/SPRING. Comp. Phys. Commun 88, 309-326 (1995)

75. F. Yuasa, D. Perret-Gallix, S. Kawabata, T. Ishikawa, Pvm-grace. Nucl. Instrum. Methods A 389, 77-80 (1997)

76. Khoze, V.A., Martin, A.D., Ryskin, M.G.: Prospects for new physics observations in diffractive processes at the LHC and tevatron. Eur. Phys. J. C 23, 311 (2002). hep-ph/0111078

77. Ingelman, Schlein, Phys. Lett. B152, 256 (1985)

78. Aktas, A., et al., (H1 Collaboration), Measurement and QCD analysis of the diffractive deep-inelastic scattering cross-section at HERA, Eur. Phys. J. C 48, 715 (2006). hep-ex/0606004

79. Aktas, A., et al.: H1 Collaboration. Dijet cross sections and parton densities in diffractive DIS at HERA, JHEP. 0710, 042 (2007). arXiv:0708.3217 